\title{
The use of fentanyl buccal tablets for breakthrough pain by using doses proportional to opioid basal regimen in a home care setting
}

\author{
Sebastiano Mercadante - Giampiero Porzio • \\ Federica Aielli • Lucilla Averna • Corrado Ficorella • \\ Alessandra Casuccio
}

Received: 30 August 2012 / Accepted: 25 March 2013 /Published online: 7 April 2013

(C) Springer-Verlag Berlin Heidelberg 2013

\begin{abstract}
The dose of rapid onset opioids to be given for breakthrough cancer pain (BTcP) is controversial. Dose proportional to the basal opioid regimen seem to be safe and effective in hospital units. However, data in other less protected settings, like home care, are lacking. The aim of this open-label study was to assess the efficacy and safety in a group of patients with BTcP followed at home, after giving a dose of fentanyl buccal tablets (FBT) proportional to the opioid basal regimen, skipping the steps for dose titration. Consecutive patients admitted to a home care program presenting BTcP episodes and receiving stable doses of opioids for background pain were selected. Data from four consecutive episodes of BTcP were collected. For each episode, patients were instructed to routinely collect changes in pain intensity and severe adverse effects when pain got severe (T0)
\end{abstract}

S. Mercadante $(\bowtie)$

Anesthesia and Intensive Care Unit \& Pain Relief and Palliative

Care Unit, La Maddalena Cancer Center, Via S.Lorenzo 312,

90145 Palermo, Italy

e-mail: terapiadeldolore@lamaddalenanet.it

S. Mercadante

e-mail: 03sebelle@gmail.com

S. Mercadante

Department of Anesthesiology, Intensive care and Emergency, Palliative Medicine Teaching, University of Palermo, Palermo, Italy

G. Porzio $\cdot$ F. Aielli $\cdot$ L. Averna $\cdot$ C. Ficorella

Department of Oncology, University of L'Aquila and L'Aquila per la vita — home care, L'Aquila, Italy

A. Casuccio

Department of Experimental Biomedicine and Clinical

Neuroscience, University of Palermo, Palermo, Italy and to reassess the same items 15 min after FBT, given as a rescue medication in doses proportional to the daily opioid doses used for background pain (T15). One hundred twenty episodes of BTcP were recorded in 30 patients. One hundred eight episodes were defined as successfully treated, while 12 episodes required a further administration of opioids. Pain intensity significantly decreased at T15 $(p<0.001)$. In 95.5 and $90.8 \%$ of episodes treated, there was a reduction in pain intensity of more than 33 and $50 \%$, respectively. No relevant adverse effects were recorded, even in older patients. This study suggests that FBT given in doses proportional to the basal opioid regimen for the management of BTcP is very effective and safe in clinical practice in the home care setting.

Keywords Cancer pain · Breakthrough-episodic pain · Fentanyl buccal tablet · Opioids $\cdot$ Home care

\section{Introduction}

In the cancer population, breakthrough pain (BTP) is a transitory exacerbation of pain, severe in intensity and with a rapid onset, superimposed on an otherwise stable pain pattern in patients treated with opioids $[9,23]$. The presence of BTP has been considered as a negative prognostic factor and infers the quality of life of these patients [24]. The availability of supplemental doses of opioids (rescue medication) in addition to the continuous analgesic medication is the main treatment suggested to manage these pain flares [10]. For years, anecdotal experience has suggested that the starting effective dose of BTP medication must be a percentage of a patient's total daily opioid dose [6]. As pain relief is usually required urgently in the last decade, new 
routes of administration have been designed to provide fast pain relief. The use of these fentanyl delivery systems, commonly named rapid onset opioids (ROOs), has been shown to provide the best effective treatment in comparison with placebo or oral morphine $[5,11]$. All the trials with ROOs, including oral transmucosal fentanyl citrate (OTFC) and fentanyl buccal tablets (FBT), suggest to titrate the doses of fentanyl to achieve the effective dose, as there is no relationship between the effective fentanyl dose and a fixed schedule opioid regimen [2, 5, 9, 27]. However, the evidence is only indirect because it is derived from the study design rather than on convincing comparative studies of dosing strategies. In these regulatory studies, a substantial proportion of patients failed the dose titration of OTFC or FBT. Moreover, an unclear distinction between the basal pain of mild-moderate intensity and BTP of moderatesevere intensity makes the interpretation of data provided by these studies difficult $[13,15]$. Observations from data pooled from trials of OTFC showed a statistically significant relationship between the breakthrough dose and around-theclock dose, despite an enormous interindividual variability in patients' dose requirements for BTP [7].

Indeed, the reasons for these findings have not been clearly explained, considering that the presence of tolerance should suggest a dose proportional to those used for background analgesia for obtaining an effect. An expected tolerance to adverse effects in patients chronically exposed to opioids has been found, despite serum fentanyl levels as high as 6$8 \mathrm{ng} / \mathrm{mL}$ [1]. Dose titration may make the practical use of ROOs difficult in the daily activity, particularly at home or in outpatients. Moreover, using different strengths of ROOs for treating each episode may be time consuming, exceeding the spontaneous duration for BTP which can spontaneously subside, as evidenced by successful placebo-treated patients [13, 15]. Finally, many patients may be reluctant to try the dose and avoid the use of these drugs, preferring, at the end, traditional oral dosing of morphine [4]. In a recent survey, most patients were using oral opioids, and only $55 \%$ of patients took rescue medication every time they experienced BTP [6]. Thus, low doses of ROOs, started in an attempt to titrate the doses individually, could result in unnecessary suffering, lowering of clinical compliance, and refusal to continue the treatment, particularly in specific settings, such as palliative home care or outpatient clinics, where patients should try the doses with different attempts.

The opioid dose to be administered for BTP remains controversial. A predictable dose could favor an easy prescription resulting in a better patients' compliance. The use of proportional doses has been shown to be safe and effective in a large number of patients in several open-label and controlled studies [16-20]. FBT given even at high doses proportional to the opioid doses used for background analgesia was safe and effective [21]. This data was explained by the high level of tolerance achieved by patients receiving high doses of opioids for background analgesia. In the only existing comparative study, this approach has been found to be more effective than dose titration method [14].

However, data on the use of proportional dose of FBT at home, where patients are more frequently assisted during the course of disease, are lacking. The aim of this study was to prospectively assess the efficacy and safety of FBT in doses proportional to opioid doses for background analgesia given chronically for the treatment of BTP of cancer patients followed at home by a palliative care team.

\section{Methods}

A cohort of patients consecutively admitted to a home care program, L'Aquila per la vita, in a period of 6 months, from June 2011 to February 2012, was surveyed.

Patients with relevant changes in renal and liver function as well as patients with cognitive disturbances or not collaborative were excluded. In addition, patients who had unstable background analgesia or more than four episodes of BTP/day were excluded. The criterion to define BTP was reported in previous studies $[6,12,14,21]$. From this sample, patients who were receiving opioids in doses of oral morphine equivalents equal or more than $60 \mathrm{mg}$ daily were prescribed FBT in doses proportional to opioids used for background analgesia. For example, the minimal existing dose, $100 \mu \mathrm{g}$ was given to patients receiving $60 \mathrm{mg}$ of oral morphine equivalents, $200 \mu \mathrm{g}$ was given to patients receiving $120 \mathrm{mg}$ of oral morphine equivalents, $300 \mu \mathrm{g}$ for $180 \mathrm{mg}$ of oral morphine equivalents, and so on. Intermediate doses were rounded at the lower dose strength (example $200 \mu \mathrm{g}$ for $150 \mathrm{mg}$ of oral morphine equivalents). Informed consent and institutional approval were obtained.

Patients were treated according to a routine protocol. After establishing effective around-the-clock opioid medication, according to opioid titration process, achieving a stable analgesia, with mean pain intensity equal or less than $4 / 10$, for two consecutive days, patients were instructed to administer FBT at the doses calculated when their pain gets severe, as superimposed episode of BTP occurs. Patients and relatives were trained in using FBT, explaining the characteristics and the way to use it.

For each episode of BTP, patients and their relatives recorded in a specific sheet the pain intensity (numerical scale $0-10$ ) and adverse effect intensity, using a scale from 0 to 3 (absent, slight, moderate, severe), and the occurrence of adverse effects considered severe enough in intensity to require a medical intervention just before starting the FBT dose (T0) and 15 min after (T15). The administration of BTP opioid medication was considered unsuccessful whenever a further BTP medication was required in the subsequent $2 \mathrm{~h}$. An on 
call service is offered for any occurrence, other than home care routine visits planned about three times a week for both physicians and nurses.

The principal outcome was the evaluation of the number of patients who benefitted from FBT (at least $>33 \%$ of decrease in pain intensity) using doses proportional to the basal opioid regimen, within $15 \mathrm{~min}$, which was considered as a clinically meaningful time to evaluate a dose administered as needed and the occurrence of adverse effects severe enough in intensity to require a medical intervention. The secondary outcome was to evaluate this aspect in older patients, which should be considered the most susceptible population to opioid analgesics.

\section{Statistical analysis}

One hundred twenty episodes treated with FBT at proportional doses to opioid doses used for background analgesia were deemed to be sufficient for an analysis, according to numbers previously published in open-label studies. For analysis, patients were divided according to the age: $\leq 65$ and $>65$ years. Frequency analysis was performed using the chi-square test. Frequency analysis was performed using the Pearson's chi-square test and Fisher's exact test. The oneway analysis of variance and Kruskal-Wallis statistic test were used to compare the different parametric or nonparametric variables. Data was analyzed by SPSS Software 14.0 version (SPSS, Inc., Chicago, Ill, US). All $P$ values were two-sided, and $P$ values less than 0.05 were considered statistically significant.

\section{Results}

Thirty patients were surveyed. The mean age was 68.9 years $( \pm 13.6)$, and $19(63.3 \%)$ of them were over 65 years.
Sixteen patients were male. One hundred twenty episodes of BTP were recorded (four for each patient). Of the 120 valuable episodes, a further BTP medication was required in the subsequent $2 \mathrm{~h}$ in 12 cases $(10 \%)$. The mean dose of FBT administered was $266.7 \mu \mathrm{g}( \pm 209.9)$, with 44 episodes $(36.7 \%)$ treated with doses of FBT $>200 \mu \mathrm{g}$. Older patients received lower doses of opioids for background analgesia and, as a consequence, lower doses of FBT $(p<0.002)$ (see Table 1).

Pain intensity significantly decreased at T15 $(p<0.0005)$ (see Table 1).In 117 (97.5\%) and 109 (90.8\%) episodes, there was a reduction in pain intensity at T15 of more than 33 and $50 \%$, respectively. The number of episodes with a decrease in pain intensity of more than $33 \%$ was higher in females $(p<0.035)$. This data was not significant for a decrease in pain intensity of more than $50 \%(p<0.112)$. Changes in pain intensity were not significant according to the age ( $p=0.758$ and $p=0.117$, respectively). No adverse effects severe enough in intensity to require medical intervention were observed. No age-related differences in adverse effects intensity were found.

\section{Discussion}

The data of this study suggest that FBT, used in proportional doses to the opioid basal regimen for the management of BTP, is effective and safe, even at home, whereas dose titration may be problematic, particularly in older patients. These findings confirm preliminary observations in patients admitted to hospital units who were receiving high doses of opioids for background analgesia and who were effectively treated with proportional doses of FBT, without adding risks of occurrence of adverse effects [21]. As this approach could not be generalized due to the characteristics of the unit, this hypothesis was tested in another setting, like home care.

Table 1 Characteristics of patients, doses of FBT, number of BTcP episodes with a decrease in PI $>33$ and $50 \%$, respectively, and number of episodes treated resulting in adverse effect intensity of mild-moderate intensity

\begin{tabular}{|c|c|c|c|c|}
\hline & All & Age $\leq 65$ years & Age $>65$ years & $P$ value \\
\hline Number of patients & 30 & 11 & 19 & \\
\hline $\mathrm{M} / \mathrm{F}$ & & 16 & 14 & \\
\hline Age & $68.9(13.6)$ & $53.8(7.5)$ & $77.7(6.6)$ & \\
\hline Evaluable episodes & 120 & 44 & 76 & \\
\hline Mean doses of FBT, $\mu \mathrm{g}(\mathrm{SD})$ & $266.7(209.9)$ & $350(235)$ & $218(179)$ & 0.002 \\
\hline T0 pain intensity & $8.7(0.8)$ & $9.0(0.9)$ & $8.6(0.7)$ & 0.002 \\
\hline T15 Pain intensity & $3.0(1.4)$ & $3.4(1.9)$ & $2.8(0.9)$ & 0.071 \\
\hline Number of BTcP episodes with a decrease in PI $>33 \%$ & 117 & 41 & 76 & $0.047^{\mathrm{a}}$ \\
\hline Number of BTcP episodes with a decrease in PI $>50 \%$ & 109 & 35 & 74 & $0.002^{\mathrm{a}}$ \\
\hline Number of BTcP episodes with AE intensity $1-2$ & 19 & 6 & 13 & $0.796^{\mathrm{a}}$ \\
\hline
\end{tabular}

${ }^{\text {a }}$ Fisher's exact test 
This observation contradicts previous indications. Data from clinical studies of FBTshow that the patients' effective doses, ranging from 100 to $800 \mu \mathrm{g}$, required a titration process [8] because of the lack of relationship between the effective fentanyl dose and a fixed schedule opioid regimen [26]. Dose titration, however, may fail, especially for patients receiving high doses of opioids for background analgesia, or may require prolonged periods of time to achieve the effective dose $[13,15]$. Of interest, in a survey reproducing a real clinical scenario, patients receiving a mean oral morphine daily dose of $132 \mathrm{mg}$ required $800 \mu \mathrm{g}$ of OTFC [26], suggesting that titration process may provide even higher doses than those expected by using proportional doses to basal opioid regimen. Moreover, observations from data pooled from trials of OTFC showed a statistically significant relationship between the breakthrough dose and around-the-clock dose, despite an enormous interindividual variability in patients' dose requirements for BTP [9].

To affirm such a paradigm, titration method should be compared with proportional doses in terms of efficacy and safety, and no study has been performed with this purpose. Data simulations have shown that doses reached after titration were quite similar to the doses calculated according the proportional paradigm [13, 15]. Indeed, titration strategy was recently compared with a traditional approach by using doses proportional to basal opioid regimen. In patients receiving daily doses of oral morphine equivalents of $>120 \mathrm{mg} /$ day, a significant number of patients obtained a decrease in pain intensity of $>50 \%$ in the proportional group in comparison with the titration group, and the need of a further rescue dose was significantly more often reported in the titration group for the first episode of BTP. This finding was not associated with differences in adverse effect intensity between the two groups [14]. In other words, proportional doses could be given more effectively, without exposing patients to more adverse effects, as suggested by several practical experiences with intravenous morphine and ROOs, even at high doses and in elderly patients [16-21].

As in a previous experience, and differently from previous controlled studies of opioid titration, the selection of BTP events to be included was strict and may allow a better interpretation of data, as patients were trained to distinguish acceptable fluctuations of pain. In this study, the mean intensity of BTP was higher and clearly distinguished from the intensity of basal pain, avoiding the influence of the gray area of mild-moderate pain for either background analgesia and BTP, often observed in these studies, which may infer the quality and interpretation of data collected. Similarly, the cut off for efficacy was more restrictive (at least a $33 \%$ decrease in pain intensity) in respect to a decrease of 2 points in a numerical scale. Despite the uncontrolled nature of the study design, the strictest selection of the BTP events to be treated offers a clinical outcome reproducible in the daily practice. The treatment was safe and effective, and only a minority of patients required a further treatment in the next $2 \mathrm{~h}$. Trained patients autoadministered the doses prescribed, according to the proportionality between dose as needed and background analgesics.

It could be argued that this approach could expose patients to adverse effects. However, no patient required a medical intervention, including older patients who could potentially be at more risk of developing adverse effects. On the other hand, when doses of FBT were titrated up to an effective mean dose of $554 \mu \mathrm{g}$ of FBT in patients who were receiving mean doses of $240 \mathrm{mg} /$ day oral morphine equivalents as background analgesia, approximately half of the patients used the maximum FBT dose of $800 \mu \mathrm{g}$ during maintenance treatment. Of interest, the effective doses of FBT were consistently higher than those eventually calculated with a proportional approach $(400 \mu \mathrm{g}$ for mean doses of $240 \mathrm{mg}$ of oral morphine equivalents as ATC analgesia); confirming that overdosing with proportional doses calculated according the opioid basal regimen is unlikely. These patients, however, afforded titration with evident suffering during the days before achieving the right dose [25].

This observation, explained by the level of opioid tolerance produced by opioid doses used for background analgesia, is a protective factor against the occurrence of severe adverse effects produced by approximately $20 \%$ of the daily dose, also explaining the high percentage of successfully treated episodes. This is confirmed by the fact that intravenous morphine, given as a bolus in doses proportional to basal opioid regimen in a thousand of episodes, has been proved to be safe and effective even at high doses and in the elderly [16-19].

Patients treated efficaciously at home may avoid hospital admissions and reduce social and economic resources. Simplifying the treatment, while providing efficacy and safety, is of paramount importance and could help both patients and professionals, as many patients have concerns in titrating doses of ROOs. In Italian surveys on attitudes of physicians on using BTP medications, this approach is used in less than $50 \%$ of patients $[3,22]$.

In conclusion, FBT given in doses proportional to the daily doses of opioids used for background analgesia was effective and safe as a breakthrough pain medication also in a home care setting. This approach was safe, as no relevant adverse effects were reported, and older patients did not present more risks of adverse effects. These results should be replayed to reinforce this concept and provide clear prescription for patients.

Acknowledgment We wish to thank the patients, relatives, and the nursing staff of L' Aquila per la Vita for the support provided in recording the data.

No conflict of interest declared.

No funding 


\section{References}

1. Aronoff G, Brennan M, Pritchard D, Ginsberg B (2005) Evidencebased oral transmucosal fentanyl citrate (OTFC) dosing guidelines. Pain Med 6:305-314

2. Caraceni A, Martini C, Zecca E, Portenoy RK et al (2004) Working group of an IASP task force on cancer pain. Breakthrough pain characteristics and syndromes in patients with cancer pain. An international survey. Palliat Med 18:177-183

3. Corli O, Pizzuto M (2011) Capire e trattare il brealkthrough cancer pain. Center for the Evaluation and Research on Pain. CIC edizioni internazonali 9-25

4. Davies A, Vriens J, Kennett A, McTeggart (2008) An observational study of oncology patients' utilization of breakthrough pain medication. J Pain Symptom Manage 35:406-411

5. Davies AD, Dickman A, Reid C, Stevens A, Zeppetella G (2009) The management of cancer-related breakthrough pain: recommendations of a task group of the science committee of the Association for Palliative Medicine of Great Britain and Ireland. Eur J Pain 13:331-338

6. Davies A, Zeppetella G, Andersen S et al (2011) Multi-centre European study of breakthrough cancer pain: pain characteristics and patient perceptions of current and potential management strategies. Eur J Pain 15:756-763

7. Hagen NA, Fisher K, Victorino C, Farrar JT (2007) A titration strategy is needed to manage breakthrough cancer pain effectively: observations from data pooled from three clinical trials. J Palliat Med 10:47-55

8. Hanks GW, De Conno F, Cherny N et al (2001) Morphine and alternative opioids in cancer pain: the EAPC recommendations. $\mathrm{Br}$ J Cancer 84:587-593

9. Kleeberg UR, Filbet M, Zeppetella G (2011) Fentanyl buccal tablet for breakthrough cancer pain: why titrate? Pain Pract 11:185-190

10. Mercadante S, Arcuri E (1998) Breakthrough pain in cancer patients: pathophysiology and treatment. Cancer Treat Rev 24:425443

11. Mercadante S (2012) Pharmacotherapy for breakthrough cancer pain. Drugs 72(2):181-190

12. Mercadante S, Ferrera P, Adile C, Casuccio A (2011) Fentanyl buccal tablets for breakthrough pain in highly tolerant cancer patients: preliminary data on the proportionality between breakthrough pain dose and background dose. J Pain Symptom Manage 42:464-469

13. Mercadante S (2011) The use of rapid onset opioids for breakthrough cancer pain: the challenge of its dosing. Crit Rev Oncol Hematol 80:460-465
14. Mercadante S, Gatti A, Porzio G, Lo Presti C, Aielli F, Adile C, Casuccio A (2012) Dosing fentanyl buccal tablet for breakthrough cancer pain: dose titration versus proportional doses. Curr Med Res Opin 28:963-968

15. Mercadante S (2009) Breakthrough pain: on the road again. Eur J Pain 13:329-430

16. Mercadante S (2010) Intravenous morphine for management of cancer pain. Lancet Oncol 11:484-489

17. Mercadante S, Villari P, Ferrera P, Bianchi M, Casuccio A (2004) Safety and effectiveness of intravenous morphine for episodicbreakthrough pain, using a fixed ratio with the oral daily morphine dose. J Pain Symptom Manage 27:352-359

18. Mercadante S, Intravaia G, Villari P, Ferrera P, Riina S, Mangione $S$ (2008) Intravenous morphine for episodic-breakthrough pain in an acute palliative care unit: a confirmatory study. J Pain Symptom Manage 35:307-313

19. Mercadante S, Villari P, Ferrera P, Casuccio A, Mangione S, Intravaia G (2007) Transmucosal fentanyl vs. intravenous morphine in doses proportional to basal opioid regimen for episodicbreakthrough pain. Br J Cancer 96:1828-1833

20. Mercadante S, Villari P, Ferrera P, Mangione S, Casuccio A (2010) The use of opioids for breakthrough pain in an acute palliative care unit by using doses proportional to opioid basal regimen. Clin $\mathrm{J}$ Pain 26:306-309

21. Mercadante S, Ferrera P, Adile C, Casuccio A (2011) Fentanyl buccal tablets for breakthrough pain in highly tolerant cancer patients: preliminary data on the proportionality between breakthrough pain dose and background dose. J Pain Symptom Manage 42:464-469

22. Mercadante S, Villari P, Casuccio A (2011) An Italian survey on the attitudes in treating breakthrough cancer pain in hospice. Support Care Cancer 19:979-983

23. Portenoy RK, Hagen NA (1990) Breakthrough pain: definition, prevalence, and characteristics. Pain 41:273-281

24. Portenoy RK, Payne D, Jacobson P (1999) Breakthrough pain: characteristics and impact in patients with cancer pain. Pain 81:129-134

25. Slatkin NE, Xie F, Messina J, Segal TJ (2007) Fentanyl buccal tablet for relief of breakthrough pain in opioid-tolerant patients with cancer-related chronic pain. J Support Oncol 5:327-234

26. Zeppetella GB (2008) Opioids for cancer breakthrough pain: a pilot study reporting patient assessment of time to meaningful pain relief. J Pain Symptom Manage 35:563-567

27. Zeppetella G, Messina J, Xie F, Slatkin NE (2010) Consistent and clinically relevant effects with fentanyl buccal tablet in the treatment of patients receiving maintenance opioid therapy and experiencing cancer-related breakthrough pain. Pain Pract 10:287-293 